\title{
Robust Output High-Gain Feedback Controllers for the Atomic Force Microscope under High Data Sampling Rate
}

\author{
Su-Hau Hsu ${ }^{1}$ and $\mathrm{Li}-\mathrm{Chen} \mathrm{Fu}^{\mathrm{l}, 2}$ \\ ${ }^{\prime}$ Department of Electrical Engineering \\ ${ }^{2}$ Department of Computer Science \& Information Engineering \\ National Taiwan University, Taipei, Taiwan, R. O. C. \\ e-mail: lichen@csie.ntu.edu.tw
}

Keywords: Atomic force microscope, nonlinear system, high-gain feedback, robust control.

\begin{abstract}
This paper proposed a robust output feedback controller of an atomic force microscope (AFM) device for the purpose of high rate date sampling. The AFM device is modeled as a cantilever-sample system in which the interactive forces between the cantilever and the sample consists of a long range attractive force and short range repulsive force. By using the feedback linearization and the singular perturbation technique, an output high-gain feedback controller is designed such that the cantilever tip can track the surface of the sample at a high rate of date sampling even though the topology of the surface is arbitrary and not given a priori. By adopting the controller developed here, the signals to be measured are the deflection of the cantilever. Finally a computer simulation is provided to demonstrate the effectiveness of the proposed controller.
\end{abstract}

\section{Introduction}

The surface topology can be probed with good atomic level accuracy using the atomic force microscope (AFM) device which was invented in 1986 [1]. A typical AFM consists of a microcantilever, a sample positioning sample system, a detection system and a control system. Sample position is provided by a piezoelectric actuator which can position the sample in two lateral directions and one vertical direction. As the sample is brought near the cantilever tip, the influence of the sample on the cantilever becomes significant enough to be registered by the detection system. A popular detection technique is based on the optical lever method where the laser incident on the top surface of the cantilever is deflected into a split photodiode sensor. The general construction is shown in Fig. 1. What is crucial in the operation described above is the cantilever, which largely determines the achievable sensitivity and resolution of the AFM. Due to the nonlinear interactive force between the cantilever and the sample, the dynamic model of the AFM becomes nonlinear one. It has been experimentally observed that the motion of the cantilever can be chaotic under certain physical condition [2]. This type of irregular motion is highly undesirable for the AFM performance since it results in inaccurate measurements. Salapaka et al. [3] showed that the amplitude and sine of the phase of the orbit appear ra- ther linear with respect to the distance between the cantilever beam and the sample. Ashhab et al. [4] have, theoretically, studied the modeling and control of the AFM tip.

This paper is concerned with the robust output feedback control of an atomic forces microscope (AFM) device in the operation for high rate data sampling. In such a device, the cantilever-sample forces are composed of a long range attractive force and a short range repulsive force. Applying the feedback linearization and the singular perturbation technique, we guarantee that the proposed an output feedback high-gain controller can drive the cantilever tip to track the surface of the sample at a high data sample rate. Despite that the topology of the surface is arbitrary and is not known a priori, the cantilever tip can succeed in tracking task subject to only very little error. During the operation, all what we need to measure is just the deflection of the cantilever. Finally, a computer simulation is made to illustrate the controller performance.

\section{Problem Statement}

The interaction potential for the cantilever-sample system is derived in [5]. According to the tip specification in Table I, the potential formula is found as follows

$$
U_{m}=-\frac{A}{d_{t s}^{\mathrm{t}}}+\frac{B}{d_{\omega^{\prime}}^{7}}
$$

where the values of $A$ and $B$ are $1.667 \times 10^{-28} \mathrm{Kg} \mathrm{m}^{3} / \mathrm{sec}^{2}$ and $2.381 \times 10^{-85} \mathrm{Kg} \mathrm{m}^{9} / \mathrm{sec}^{2}$, respectively, $d_{s s}$ is the distance between the cantilever tip and the surface of the sample. The cantilever-sample forces are composed of a long range attractive force and a short range repulsive force, and the resulting force-distance diagram is shown in Fig 2.

The coordinate of the cantilever-sample system is shown in Fig. 3. Let $\phi_{f i}(x), i=1,2, \ldots$, be the assumed mode shape functions pertaining to the cantilever beam. Let the cantilever beam be of length $L$ with a uniform density $\rho$, and constant flexural rigidity $E I$. Let the sample be of mass $M_{D}$. Here, the cantilever beam is considered as a single-link flexible manipulator. Generally, it is difficult to find the natural modes of the beam. However, we can use some 
appropriate assumed mode shapes to estimate the beam behavior [5]. In our case here, the cantilever beam is an Euler-Bernoulli beam subject to a clamped-free boundary condition. After calculating the total kinetic energy and the potential energy of the cantilever-sample system, we establish the dynamic model by substituting the Lagrangian, the total kinetic energy minus the total potential energy, into the Lagrangian equations. The total kinetic energy consists of the kinetic energy of the cantilever beam and the kinetic energy of the sample. On the other hand, the total potential energy consists of both the elastic strain energy in the beam and the cantilever-sample potential. Now, if only first mode is considered, the dynamic model can be derived as follows

$$
\begin{aligned}
\dot{x}_{1} & =x_{2} \\
\dot{x}_{2} & =\frac{a_{21}}{\left(\left.x_{1} \phi_{1}\right|_{l=L}+d_{0}-x_{3}-d_{p}\right)^{2}}+\frac{a_{22}}{\left(\left.x_{1} \phi_{1}\right|_{l=L}+d_{0}-x_{3}-d_{p}\right)^{8}} \\
& +a_{23} x_{1} \\
\dot{x}_{3}= & x_{4} \\
\dot{x}_{4} & =\frac{a_{41}}{\left(\left.x_{1} \phi_{1}\right|_{l=L}+d_{0}-x_{3}-d_{p}\right)^{2}}+\frac{a_{42}}{\left(\left.x_{1} \phi_{1}\right|_{l=L}+d_{0}-x_{3}-d_{p}\right)^{8}} \\
& +a_{43} F
\end{aligned}
$$

where

$$
\begin{array}{lll}
a_{21}=\frac{\left.A \phi_{1}\right|_{l=L}}{\rho A \int_{0}^{L} \phi_{1}^{2} d l}, & a_{22}=\frac{\left.B \phi_{1}\right|_{l=L}}{\rho A \int_{0}^{L} \phi_{1}^{2} d l}, & a_{23}=-\frac{E I \int_{0}^{L} \phi_{1}^{\prime \prime} d l}{\rho A \int_{0}^{L} \phi_{1}^{2} d l}, \\
a_{41}=\frac{\left.A \phi_{1}\right|_{l=L}}{M_{D}}, & a_{42}=\frac{\left.B \phi_{1}\right|_{l=L}}{M_{D}}, & a_{43}=\frac{1}{M_{D}}
\end{array}
$$

In the model above, $x_{1}$ the first flexible mode of the cantilever, $x_{3}$ is the displacement of the sample, $F$ is the force applied on the sample from the piezoelectric actuator, $d_{0}$ is the initial gap between the tip and the surface of the sample, and $d_{p}$ is the variation of the surface of the sample which is assumed to be smooth (i.e., $C^{\infty}$ ). For convenience, we define the errors $e_{1}, e_{2}$ as

$$
e_{1}=x_{1}, \quad e_{3}=x_{3}+d_{p}
$$

and rewrite the original dynamics as

$$
\begin{aligned}
& \dot{e}_{1}=e_{2} \\
& \dot{e}_{2}=\frac{a_{21}}{\left(\left.e_{1} \phi_{1}\right|_{l=L}+d_{0}-e_{3}\right)^{2}}+\frac{a_{22}}{\left(\left.e_{1} \phi_{1}\right|_{l=L}+d_{0}-e_{3}\right)^{8}}+a_{23} e_{1} \\
& \dot{e}_{3}=e_{4} \\
& \dot{e}_{4}=\frac{a_{41}}{\left(\left.e_{1} \phi_{1}\right|_{l=L}+d_{0}-e_{3}\right)^{2}}+\frac{a_{42}}{\left(\left.e_{1} \phi_{1}\right|_{l=L}+d_{0}-e_{3}\right)^{8}}+a_{43} F+\ddot{d}_{p}
\end{aligned}
$$

which is then termed as the error dynamics. Our goal is twofold: to make the tip track the surface of the sample, and to suppress the vibration on the cantilever beam, i.e., to drive $e_{1}$ and $e_{3}$ to converge to zero. Form the error dynamics, it is apparent that the variation of the surface of the sample is just like a disturbance. When its amplitude is larger and its frequency is higher, the disturbance is stronger. In practical applications, we can not know the disturbance a priori, and, furthermore as we mentioned in the introduction, the only signal we can measure is nothing else but the deflection of the cantilever. Hence, in order to make $e_{1}$ and $e_{3}$ converge, a robust output feedback controller has to be developed. In the next section, we will design such a controller and guarantee its stability property.

\section{Robust Output Feedback Controller}

\subsection{Controller Design}

Consider a nonlinear single input, single output system of the form

$$
\begin{aligned}
& \dot{x}=f(x)+g(x) u+p(x) w \\
& y=h(x)
\end{aligned}
$$

The first equation of this system describes a plant with state $x$, defined over a neighborhood $D_{x}$ of the origin in $R^{n}$, with control input $u \in R^{\prime}$, and subject to a disturbance $w \in R^{1}$. The second one defines a measured variable $y \in R^{\prime}$, which is a function of state plane $x$. The functions $f(x), g(x), p(x)$ are smooth mappings defined over some neighborhood of the origin in $R^{n}$. The control objective here is to develop provide a controller, which processes the measured variable $y$, generates the appropriate control input $u$, and bears the following structure

$$
\begin{aligned}
& \dot{\xi}=\eta(\xi, y) \\
& u=\theta(\xi)
\end{aligned}
$$

in which $\xi$ is defined over a neighborhood $D_{\xi}$ of the origin in $R^{\nu}$ and $\eta: D_{\xi} \times R^{\prime} \rightarrow R^{n}, \theta: D_{\xi} \rightarrow R^{1}$ are $C^{\infty}$ functions, satisfying $\eta(0,0)=0, \theta(0)=0$. The following theorem states the conditions under which the hereby developed controller can stabilize the overall system.

Theorem 3.1. If the system (3-1) satisfies the following condition:

(i) $L_{g} L_{f}^{k} h(x)=0$ for all $x$ in a neighborhood of $x=0$ and for all $k=0,1, \ldots,(n-2)$,

(ii) $L_{g} L_{f}^{n-1} h(0) \neq 0$,

(iii) $L_{p} L_{f}^{k} h(x)=0$ for all $x$ in a neighborhood of $x=0$ and for all $k=0,1, \ldots,(n-2)$,

(iv) $L_{p} L_{f}^{n-1} h(0) \neq 0$,

(v) all the roots of the polynomial equations

$$
n(s)=s^{n-1}+c_{n-2} s^{n-2}+\cdots+c_{1} s^{1}+c_{0}=0,
$$

have negative real parts, 
then there exists a controller as described by

$$
u=-K\left[y^{(n-1)}(t)+c_{n-2} y^{(n-2)}(t)+\cdots+c_{1} y(t)\right]
$$

which stabilizes the system (3-1), provided $K$ is a suffciently large constant with the same sign as that of $L_{g} L_{f}^{n-1} h(0)$.

Proof. According to the feedback linearization, with Condition (i) (iv), clearly there exists a coordinate transformation $z=\Phi(x)$ as follows

$$
\begin{aligned}
z_{1} & =\Phi_{1}(x)=h(x)=y \\
z_{2} & =\Phi_{2}(x)=L f h(x)=y^{(1)} \\
& \cdots \\
z_{n} & =\Phi_{n}(x)=L_{f}^{n-1} h(x)=y^{(n-1)}
\end{aligned}
$$

such that the state-space description of the system (3-1) in this new coordinates will be as follows

$$
\begin{aligned}
\dot{z}_{1} & =z_{2} \\
\dot{z}_{2} & =z_{3} \\
& \cdots \\
\dot{z}_{n-1} & =z_{n} \\
\dot{z}_{n} & =L_{f}^{n} h\left[\Phi^{-1}(z)\right]+L_{g} L_{f}^{n-1} h\left[\Phi^{-1}(z)\right] u \\
& +L_{\rho} L_{f}^{n-1} h\left[\Phi^{-1}(z)\right] w
\end{aligned}
$$

Suppose $L_{g} L_{f}^{n-1} h(0)>0$ (the other case can be dealt with in the same manner). Set $K=1 / \varepsilon$ and substituting the control law into the system, the system becomes

$$
\begin{aligned}
\dot{z}_{1} & =z_{2} \\
\dot{z}_{2}= & z_{3} \\
& \cdots \\
\dot{z}_{n-1} & =z_{n} \\
\varepsilon \dot{z}_{n}= & \varepsilon L_{f}^{n} h\left[\Phi^{-1}(z)\right]-L_{g} L_{f}^{n-1} h\left[\Phi^{-1}(z)\right]\left(z_{n}+c_{n-1} z_{n-1}\right. \\
& \left.+\cdots+c_{1} z_{1}\right)+\varepsilon L_{p} L_{f}^{n-1} h\left[\Phi^{-1}(z)\right] w
\end{aligned}
$$

Since $K$ is a sufficiently large constant, $\varepsilon$ is a sufficiently small constant and we can obtain the reduced subsystem

$$
\begin{aligned}
\dot{z}_{1} & =z_{2} \\
\dot{z}_{2} & =z_{3} \\
& \cdots \\
\dot{z}_{n-1} & =z_{n} \\
0 & =z_{n}+c_{n-1} z_{n-1}+\cdots+c_{1} z_{1}
\end{aligned}
$$

and the boundary-layer subsystem

$$
\frac{d y}{d \tau}=-\left\{L_{g} L_{f}^{n-1} h\left[\Phi^{-1}(z)\right]\right\} y
$$

Since Condition (v) is valid, the reduced subsystem is asymptotically stable. On the other hand, the boundary-layer subsystem is obviously asymptotically stable. Therefore, according to the singular perturbation theory [8], for sufficiently small positive $\varepsilon$, or sufficiently large $K$, the system is asymptotically stable.

Clearly the controller in Theorem 3.1 is not physically realizable, but it is not difficult to replace it by a suitable physically realizable approximation, without impairing the stability property of the corresponding closed-loop system.

Lemma 3.2.[9] Suppose the system

$$
\dot{x}=f(x)-g(x) k(x) K
$$

is asymptotically stable at the equilibrium $x=0$. Then, if $T$ is a sufficiently small positive number, also the system

$$
\begin{aligned}
& \dot{x}=f(x)-g(x) \xi \\
& \dot{\xi}=\frac{1}{T}(-\xi+k(x) K)
\end{aligned}
$$

is asymptotically stable at $(x, \xi)=(0,0)$.

Note that the system discussed in this lemma is nothing else than the system to be closed with a linear system described by the transfer function

$$
H(s)=\frac{-K}{1+T s}
$$

Thus we may interpret this result as the fact that the introduction of a "small time constant" in a stable control loop does not impair (as least locally) its asymptotic stability. Using this lemma $r-1$ times, we can deduce that there exists a high-order filter without impairing the asymptotic stability of the original system. Hence, we obtain the following main theorem of this paper.

Theorem 3.3. If the system (3-1) satisfies the following conditions:

(i) $L_{g} L_{f}^{k} h(x)=0$ for all $x$ in a neighborhood of $x=0$ and for all $k=0,1, \ldots,(n-2)$,

(ii) $L_{g} L_{f}^{n-1} h(0) \neq 0$,

(iii) $L_{p} L_{f}^{k} h(x)=0$ for all $x$ in a neighborhood of $x=0$ and for all $k=0,1, \ldots,(n-2)$,

(iv) $L_{p} L_{f}^{n-1} h(0) \neq 0$,

(v) all the roots of the polynomial equation (3-3) have negative real parts,

then a linear dynamic output feedback controller described by the following transfer function

$$
H(s)=\frac{-K n(s)}{(1+T s)^{n-1}}
$$

stabilizes the system, provided $K$ is a suitably large constant with the same sign as that of $L_{g} L_{f}^{n-1} h(0)$ and $T$ is a sufficiently small positive constant.

Proof. For Condition (i)-(iv), from Theorem 3.1, we know 
that there exists a controller (3-4). Using Lemma 3.2 , we can deduce that there exists a first-order filter which can be introduced to the controller $(3-4)$ without impairing the asymptotic stability of the original system, i.e., the following is a stabilizing controller sufficiently small $T$,

$$
H_{1}(s)=\frac{-K n(s)}{1+T s}
$$

By repeating this lemma $n-1$ times, there will then exist a high-order filter to be integrated with the controller (3-4) which do not impair the asymptotic stability of the original system

$$
H(s)=\frac{-K n(s)}{(1+T s)^{n-1}}
$$

for sufficiently small $T$. This completes the proof.

The following Corollary 3.4 is a formal statement of applying the technique in Theorem 3.3 to our AFM robust output feedback control problem.

Corollary 3.4. Consider the AFM problem depicted in Section 2 . We define $f(e), g(e), p(e)$ and $h(e)$ as follows:

$$
\begin{gathered}
f(e)=\left[\begin{array}{c}
\frac{a_{21}}{\left(\left.e_{1} \phi_{1}\right|_{l=L}+d_{0}-e_{3}\right)^{2}}+\frac{e_{2}}{\left(\left.e_{1} \phi_{1}\right|_{t=L}+d_{0}-e_{3}\right)^{8}}+a_{23} e_{1} \\
\frac{e_{4}}{\left(\left.e_{1} \phi_{1}\right|_{l=L}+d_{0}-e_{3}\right)^{2}}+\frac{a_{42}}{\left(\left.e_{1} \phi_{1}\right|_{l=L}+d_{0}-e_{3}\right)^{8}}
\end{array}\right], \\
g(e)=\left[\begin{array}{c}
0 \\
0 \\
0 \\
a_{43}
\end{array}\right], p(e)=\left[\begin{array}{c}
0 \\
0 \\
0 \\
-1
\end{array}\right] \text {, and } h(e)=e_{1} . \quad(3-16)
\end{gathered}
$$

If all the roots of the polynomial equation

$$
n(s)=s^{3}+c_{2} s^{2}+c_{1} s^{1}+c_{0}=0
$$

have negative real parts and $K$ is a sufficiently large constant with the same sign as that of $L_{g} L_{f}^{3} h(0)$, then, with a sufficiently small positive constant $T$, there exists a linear dynamic output feedback with transfer function

$$
H(s)=\frac{-K n(s)}{(1+T s)^{3}}
$$

which stabilizes the AFM device and achieves the tracking objective with small bounded error.

Proof. Given $f(e), g(e), p(e)$ and $h(e)$, all conditions in Theorem 3.3 are satisfied and, hence, the proof is completed.

\subsection{Simulation}

In order to illustrate the controller performance, we study a numerical case. For simulation, we want the tip to scan the sample at the speed of $1 \mathrm{~Kb} / \mathrm{sec}$; that is, the tip should read a bit per millisecond. Now we, as shown in Fig. 3, consider the variation of the surface of the sample is following a sinusoidal wave. Its amplitude is $40 \mathrm{~nm}$, and its frequency is $1 \mathrm{KHz}$. As we have mentioned in Section 2, the variation of the surface of the sample is like a disturbance and its profile with respect to the tip is shown in Fig. 4, where the zero disturbance corresponds to the distance between the tip and the sample surface kept at $d_{0}=0.44 \mathrm{~nm}$. Table I, II, and III represent the specifications of the tip, cantilever beam, and sample, respectively. The cantilever is formed with the silicon and, therefore, its modulus of elasticity and density is $190 \mathrm{Gpa}$ and $2330 \mathrm{Kg} / \mathrm{m}^{3}$, respectively [9]. With the assumed modes, we can estimate the natural angular frequency of any mode shapes. After calculation, the natural angular frequency of the first, the second, and the third mode shape are found to be about $18.7 \mathrm{kHz}, 117 \mathrm{kHz}$, and 1330 $\mathrm{kHz}$, respectively. Because the first mode frequency is much higher than $1 \mathrm{kHz}$, we can only consider the first mode and use the dynamic model in Section 2. Let the initial distance from the tip to the surface of the sample be $d_{0}=$ $0.44 \mathrm{~nm}$ and we want to regulate it at the same value even when there exists a disturbance. Figure 5 and 6 show the responses of $e_{1}$ and $e_{3}$, respectively. Figure 7 and 8 show the responses of the cantilever beam and the sample, respectively. Figure 9 shows the distance from the tip to the surface of the sample which is kept almost always about 0.44 nm. Because the errors $e_{1}$ and $e_{2}$ from Figs. 5 and 6 are so small relative to the motion range of the sample, the variation of the sample surface can be accurately decoded through the response profile of the sample from Fig. 8. As a result, the AFM device indeed plays a role the same as a microscope which simultaneously samples the date at a high rate.

\section{Conclusion}

This paper is concerned with the robust output feedback controller for a atomic force microscope (AFM) device for a high rate date sampling. The device is modeled as a nonlinear cantilever-sample system where the interactive forces are composed of a long range attractive force and a short range repulsive force. By using the feedback linearization and the singular perturbation technique, an output high-gain feedback controller is designed such that the cantilever tip can track the surface of the sample at a high sampling rate even though the topology of the surface is arbitrary and not given a priori. To implement the controller, what we need to measure is nothing but the deflection of the cantilever beam. Finally, a computer simulation is provided to demonstrate the effectiveness of our proposed controller.

\section{Bibliography}

[1] G. Binnig, and C. F. Quate, "Atomic Force Microscope", Physical Review Letter, Vol. 56, pp. 930-933, March 1986. 
[2] N. A. Burnham, A. J. Kulik, G. Gremaud, and G. A. D. Briggs, "Nanosubharmonic: The Dynamics of Small Nonlinear Contacts", Physical Review Letter, Vol. 74, pp. 5092-5059, June 1995.

[3] M. V. Salapala, D. J. Chen, J. P. Cleveland, "Stability and Sensitivity Analysis of Periodic Obits in Tapping Mode Atomic Microscopy," Proc. 37th IEEE Conf. Decision Contr., Tampa, FL, Dec. 1998, pp. 2047205.

[4] M. Ashhab, M. V. Salapaka, M. Dahleh, and I. Mezic, "Dynamical analysis and control of Micorcantilevers", Under review for Automatica, 1997.

[5] Jacob N. Israelachvili, Intermolecular and surface Forces, Academic Press, London, 1985.

[6] Anthony R. Fraser and Ron W. Daniel, Perturbation Techniques for Flexible Manipulators, Kluwer Academic Publishers, Boston, 1991.

[7] H. K. Khalil, Nonlinear Systems, Prentice-Hall, Upper Saddle River, NJ, 1995

[8] A. Isidori, Nonlinear Control Systems, SpringerVerlag, New York, 1995.

[9] W. R. Runyan, Silicon Semiconductor Technology, McGraw-Hill, New York, 1965.

Table I

Tip Specifications

\begin{tabular}{c|c}
\hline Parameters & Values \\
\hline Length & 10 micrometers \\
Radius at End & 100 Angstroms \\
Material & Silicon \\
\hline
\end{tabular}

Table II

Cantilever Specifications

\begin{tabular}{c|c}
\hline Parameters & Values \\
\hline Length & 442 micrometers \\
Width & 40 micrometers \\
Thickness & 2.5 micrometers \\
Shape & Rectangular \\
Material & Silicon \\
\hline
\end{tabular}

Table III

Sample Specifications

\begin{tabular}{c|c}
\hline Parameters & Values \\
\hline Length & $10 \mathrm{~mm}$ \\
Width & $10 \mathrm{~mm}$ \\
Average thickness & $10 \mathrm{~mm}$ \\
Shape & Rectangular \\
Material & Silicon \\
\hline
\end{tabular}

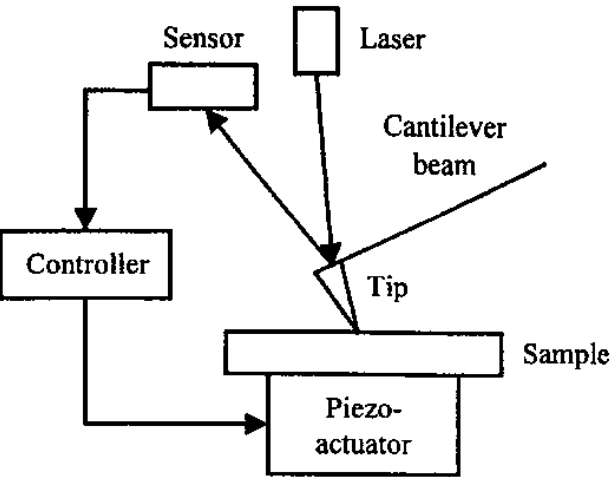

Fig1. Concept diagram of the AFM device

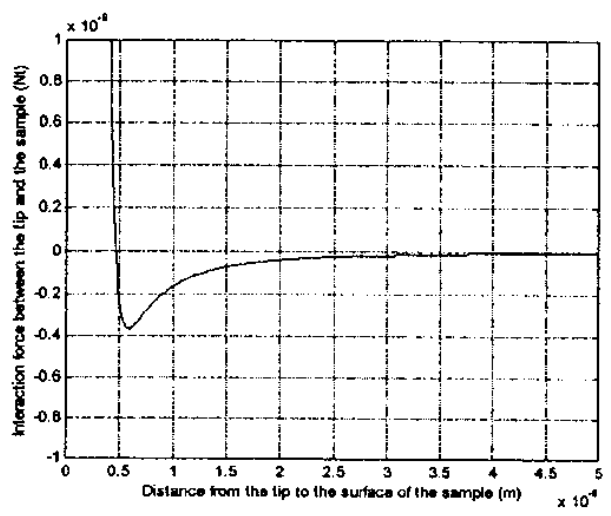

Fig.2 Force between the tip and sample

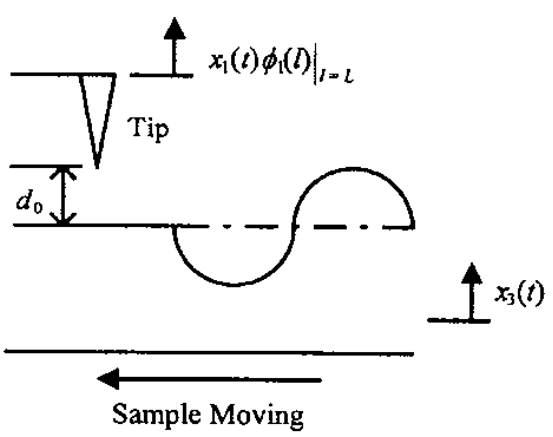

Fig.3 Coordinate diagram 


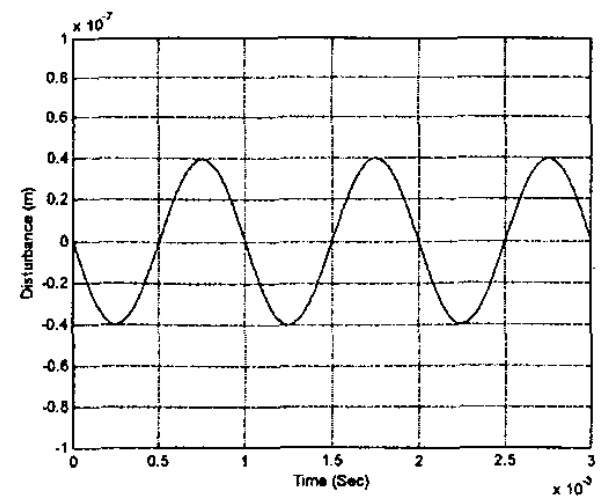

Fig4. Disturbance input $d_{p}$

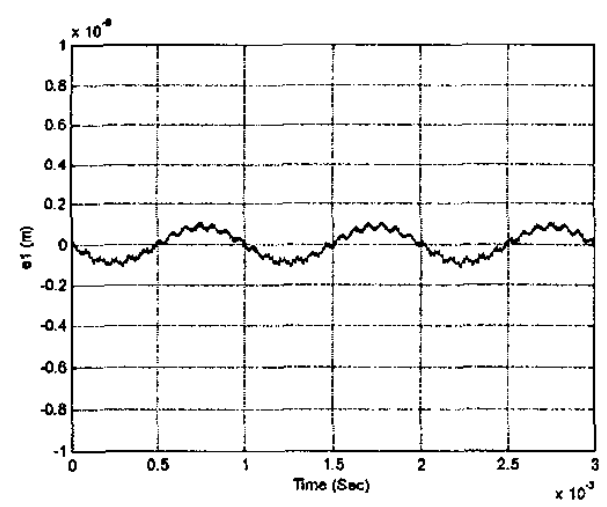

Fig.5 Response of $e_{1}$

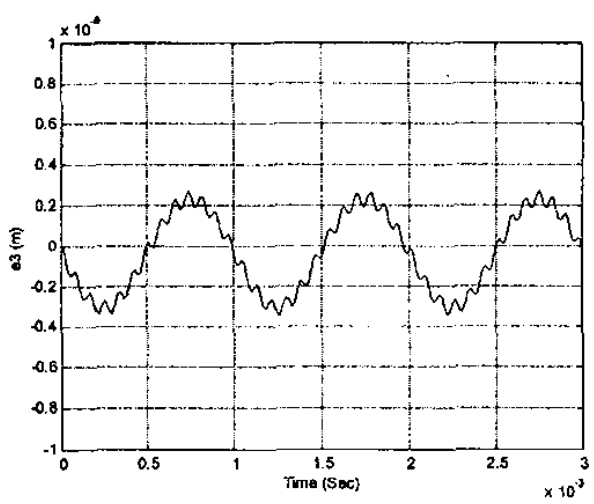

Fig.6 Response of $e_{3}$

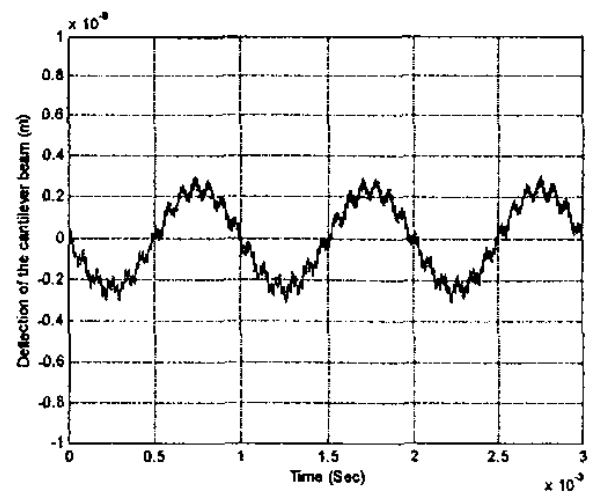

Fig.7 Response of the cantilever beam

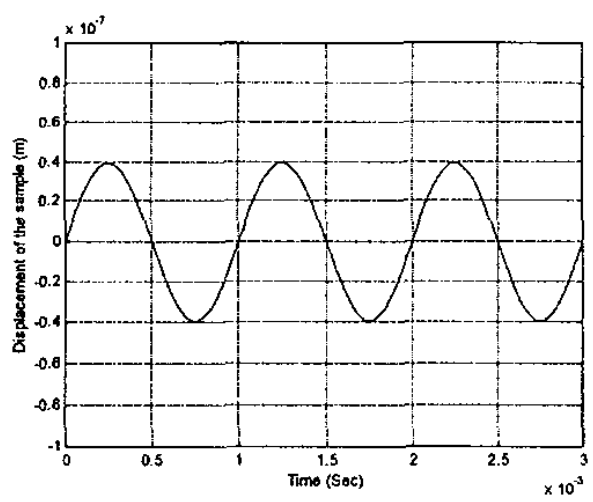

Fig. 8 Response of the sample

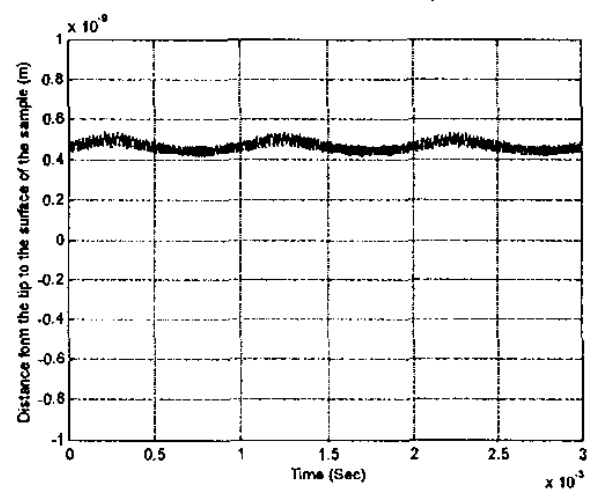

Fig.9 Distance from the tip to the surface of the sample 